\title{
THE IRAS SATELLITE - SOME REMARKS ON THE DATA, THEIR AVAILABILITY AND THEIR USE IN LEIDEN*
}

\author{
H. J. HABING \\ Sterrewacht Leiden, The Netherlands
}

(Received 27 June, 1986)

\section{About IRAS}

The Infrared Astronomical Satellite (IRAS) was jointly developed and operated by the US National Aeronautics and Space Administration (NASA), the Netherlands Agency for Aerospace Programs (NIVR), and the UK Science and Engineering Research Council (SERC). It functioned only in 1983 but it completed its main task: the making of a survey of the sky in four infrared wavelength bands. The satellite, its mission and its first results have been described elsewhere (see, e.g., the special issue of Astrophys. J. Letters, 1 March, 1984, or an article by Habing and Neugebauer in Scientific American, November 1984). In June 1985 a conference was held in Noordwijk, the Netherlands, where scientific results from the IRAS mission were discussed; the proceedings will appear in May 1986 (Israel, 1986); a second 'IRAS conference' will be held in June 1986 in Pasadena (California) and also its proceedings will be published.

The most important part of the mission was to carry out a survey of the whole sky in four broad wavelength bands, centred at $12,25,60$, and $100 \mu \mathrm{m}$, with limited angular resolution (from $0.8 \times 4.5(\operatorname{arcmin})^{2}$ at $12 \mu \mathrm{m}$ to $3.0 \times 5.0(\operatorname{arc~min})^{2}$ at $100 \mu \mathrm{m}$ ). The survey was repeated 2 times; the three surveys are called HCON1, HCON2, and HCON3 - HCON stands for 'hours confirmation': each scan was reobserved within 36 hours, to confirm detections made the first time. HCON1 observations and HCON2 observations were made usually two weeks apart; HCON3 observations were made 6 months later. Whereas HCON1 and HCON2 observations covered $95 \%$ of the sky, HCON3 was terminated at $72 \%$ completion when the last drop of coolant (superfluid $\mathrm{He}$ ) left the dewar on 22 November, 1983. Low-resolution spectra between 8 and $23 \mu \mathrm{m}$ were obtained of bright, well-isolated point sources by a separate spectrograph that operated simultaneously with the survey instrument. Since the survey operations required only $60 \%$ of the available time, the remaining $40 \%$ was used for so-called 'additional observations', in which either the survey instrument was used to do longer integrations on small parts of the sky, thus improving the signal to noise ratio, or where a special instrument, the Chopped Photometric Channel (CPC) was used to obtain

* Review presented at a Workshop on 'The Role of Dust in Dense Regions of Interstellar Matter', held at Georgenthal, G.D.R., in March 1986.

Astrophysics and Space Science 128 (1986) 157-162.

(C) 1986 by D. Reidel Publishing Company

(C) Kluwer Academic Publishers • Provided by the NASA Astrophysics Data System 
maps, with a better angular resolution at the longer wavelengths than the survey instrument provided for.

The analysis of the survey data had two different goals: (1) to provide a catalogue of infrared point sources; (2) to provide sky maps of the infrared emission. Before launch item (2) was considered a very desirable goal but one that would be probably too high; in fact the satellite performed so well in flight, that the goal could be reached quite satisfactorily. The first edition of the Point Source Catalogue appeared in November 1984, together with that of the spectra, and with the first edition of the sky maps from the HCON3 data. Successively through 1985 and 1986 further 'data products' have become available. In November 1985 all the 'additional observations' have been made public. Further processing of the original data is continuing both in the U.S. and in The Netherlands. In the U.S. a major goal is to provide a Point Source Catalogue that extends to lower sensitivity levels and to provide sky maps of better quality, both with respect to calibration and to the removal of instrumental background effects. In The Netherlands the emphasis is on improved sky maps: this is the so-called 'GEISHA' project that is caried out under supervision of Dr P. Wesselius at the University of Groningen.

Access via computer to practically all the original IRAS data and to the derived products is possible through an IRAS data centre at the Rutherford/Appleton Laboratories in the U.K. and through one at the California Institute of Technology at Pasadena (California) or through university computers at Leiden and at Groningen in The Netherlands. Several derived products, though, have been freely distributed - in continental Europa by the Centre de Données Stellaires in Strasbourg. In all cases these products are in the form of magnetic tapes; in a few cases also in hard copy. To use these distributed data with profit one needs two documents, the first describing the mission, the data analysis, and the Point Source Catalogue and the Sky Flux Maps; the second the Small-Scale Structure Catalogue. These two documents are called (1) IRAS, Catalogs and Atlases, the Expanatory Supplement and (2) IRAS Small-Scale Structures Catalog. Both documents have appeared in preprint form but they will be printed soon by the U.S. Government Printing Office. For a summary of the more current IRAS data products, see Table I.

Finally, let me give you an illustration of the IRAS results, notably a breakdown of the contents of the Point Source Catalogue; I owe this analysis to T. Chester's contribution to the 1985 IRAS conference. The Point Source Catalogue contains 158000 stars ( $65 \%$ of the total number of entries in the catalogue); 35000 'galactic objects' $(14 \%)$ : compact H II regions, hot cores of molecular clouds, planetary nebulae, etc.; 33000 $(13 \%)$ condensations in the thin 'cirrus clouds'; and $22000(9 \%)$ galaxies. Of the 158000 stars 18000 objects have colours indicating a Rayleigh-Jeans tail of a Planck curve; 81000 objects have colours indicating some circumstellar emission and 59000 stars are too faint to characterize. 
The following sets of IRAS data are present and accessible in the Netherlands. Copies of some of the sets can be obtained through the Centre de Donneées Stellaires in Strasbourg, usually at marginal costs.

The first three items are only in book form:

1. The Explanatory Supplement to the IRAS catalogs and atlases. Freely available in preprint form; only a very few copies have been left.

2. The IRAS Small-Scale Structure Catalogue - Explanatory Supplement. Freely available in preprint form.

3. Cataloged galaxies and quasars observed in the IRAS Survey. Freely available in preprint form.

The following data sets are all available on magnetic tape; sometimes a representation on microfiche or a photographic representation exists.

4. Point Source Catalogue (2 tapes; 1600 BPI). Also in microfiche. Microfiche and tape are freely available.

5. Small-Scale Structure Catalogue (1 tape; 1600 BPI). Also in microfiche. Microfiche and tape are freely available.

6. Working Survey Data Base and Ancillary File (2 tapes; 6250 BPI). This data base contains more detailed information on individual point sources from item 4, the Point Source Catalogue - e.g., individual detections. Freely available.

7. Atlas of Low Resolution Spectra (2 tapes; $6250 \mathrm{BPI}$ ). Contains $\sim 5000$ spectra between 6 and $23 \mu \mathrm{m}$ of the brighter, well-isolated point sources. Tapes are freely available. The Atlas is in press in the Supplements to Astronomy and Astrophysics.

8. Skyflux HCONI (27 tapes; $6250 \mathrm{BPI}$ ). The brightness distribution of the sky in the four wavelength bands as derived from HCON1 data, organized in plates of $16^{\circ} \times 16^{\circ}$. Freely available. A photographic copy is also available at cost price ( $\sim 1500$ Dfl.) from the Laboratory for Space Research, University of Groningen (c.o. Dr P. Wesselius).

9. Skyflux HCON3 (20 tapes; $6250 \mathrm{BPI}$ ). Freely available.

10. Zody History File 13 tapes; 1600 BPI). Brightness distribution of the sky as in the skyflux maps, but averaged over bins of $0.5^{\circ}$. Freely available.

11. Maps constructed from 'Additional Observations' taken with the CPC (3 tapes; 1600 BPI). Freely available directly from the Laboratory for Space Research, University of Groningen (c.o. Dr P. Wesselius). A special explanatory supplement is also provided.

12. Maps constructed from 'Additional Observations' taken with the 'Survey Instrument' (66 tapes; 6250 BPI). Freely available in FITS format directly from the Laboratory for Space Research, University of Groningen (c.o. Dr P. Wesselius). A special explanatory supplement is provided.

13. The full data base of the low-resolution spectrograph contains approximately 100000 spectra, most of poor quality, from which the LRS Atlas (item 7) has been constructed. This data base will not be distributed, but can be consulted in the Netherlands.

14. The CRDD data base (CRDD = Calibrated Raw Detector Data) (450 tapes; 250 BPI). Almost raw data. Not available for distribution, access is possible via Leiden University (the Observatory). Data reduction software is also available.

15. Raw detector data base (approximately 450 tapes; 6250 BPI). The raw original data. Not available for distribution, but access possible via Groningen University (Laboratory for Space Research). In a project called 'GEISHA' the data are being rearranged into an easily accessed data base and new calibration data are provided. In the future ( $>$ 1987.5?) these data sets can be quickly accessed; maps can then be derived of significantly higher quality than the present 'sky flux' products.

\section{Some Studies Based on IRAS Data and Carried out in Leiden}

IRAS data are often valuable by themselves; however, they still gain in importance if they are combined with those obtained by other astronomical instruments. Studies based on IRAS data are being carried out at each of the major astronomical research centres in The Netherlands (Amsterdam, Dwingeloo, Groningen, Utrecht, and Leiden); 
the objects range from nearby stars to 'starburst' galaxies. Here I will describe briefly a few IRAS related research topics at the Observatory in Leiden. First is the work by C. de Vries and R. S. le Poole, who discovered that the faint, extended $100 \mu \mathrm{m}$ emission, named 'Infrared Cirrus' corresponds very closely to faint emission on high-quality sky survey plates (notably the plates of the ESO/SRC southern survey). De Vries and le Poole showed that the optical emission is in fact general galactic light that is scattered back by high-latitude clouds of low extinction $\left(A_{v} \sim 0.5\right.$ to 1.5$)$. Analysis of all available data suggests that the grains have an albedo of $\sim 0.6-$ actually a confirmation of earlier results. Another conclusion is that one of the cirrus clouds is unexpectedly strong at $12 \mu \mathrm{m}$, but another is not - the difference is very significant. Excess emission at $12 \mu \mathrm{m}$ is observed in quite a few IRAS sources - however, its cause is unknown, but it may be due to very small dust particles $(<10 \mathrm{~nm})$. De Vries's thesis of May 1986 gives a full report. Second, I mention the work by E. Deul, H. Walker, and B. Burton on the zodiacal light. The zodiacal emission is a very prominent feature in all IRAS maps, but especially so in the short wavelength maps: for example galactic structure studies are seriously affected by this solar system dust. The derivation of the zodiacal light model from the data is in first instance a gigantic data processing job; it is proceeding quite well. The interpretation in terms of an ecliptic dust distribution will be done in collaboration with R. Wolstencroft from Edinburgh (and in competition with other groups in Europe and in the U.S.).

Third, surprisingly nice maps were obtained by R. Braun (Thesis, Leiden, 1985) who for the first time described the emission from several supernova remnants. If one assumes that the infrared emission is from interstellar dust, swept up by the supernova remnant (and that in the ejected gases dust has not yet formed), then the infrared radiation gives a clue to the total amount of swept up gas - a quantity that is otherwise difficult to measure. A major open question is, of course, how much of the dust has been destroyed by the supernova remnant.

Still other work involves external galaxies: P. Schwering and F. Israel have derived maps of the two Magellanic Clouds. The maps are very carefully calibrated and optimized in angular resolution. A comparison with optical and radiomaps is being made. A similar comparison is made for M31 by R. Walterbos. Schwering and Walterbos will each present their results in their theses (1987, and September 1986). More distant are the Seyfert galaxies, and other active galaxies, detected first by, but now studied by R. de Grijp, W. Keel, and G. Miley. Selecting sources from the Point Source Catalogue according to certain criteria and, next, taking optical spectra, these astronomers discovered several hundreds of previously unknown Seyfert galaxies. The work is now extended to the additional observations; galaxies are detected out to $z=0.5$.

\section{Highly Evolved Stars in the Bulge of the Galaxy}

If one selects sources from the point source catalogue with the criteria that at $12 \mu \mathrm{m}$ the flux density is below $6 \mathrm{Jy}$ and that at $25 \mu \mathrm{m}$ the flux density is the same as at $12 \mu \mathrm{m}$ 


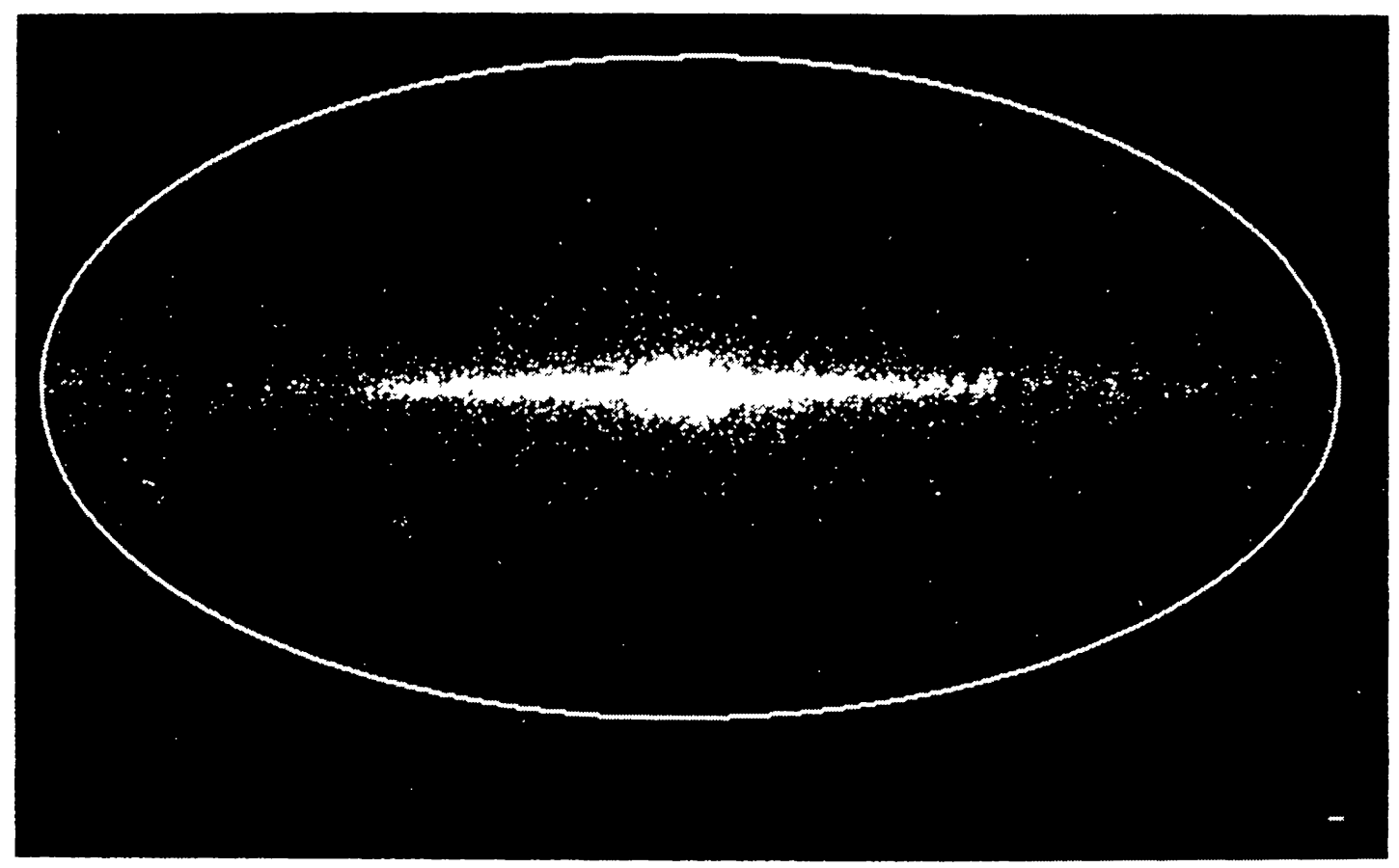

Fig. 1. Distribution on the sky of sources from the Point Source Catalogue, selected according to the criterium that the 12 and $25 \mu \mathrm{m}$ flux densities are equal within a factor of two and that the $12 \mu \mathrm{m}$ flux density is below $6 \mathrm{Jy}$.

within a factor of 2, then one derives a total sample of more than 7000 stars, distributed as in Figure 1; it shows very distinctly a disk and a bulge - clearly a spiral galaxy seen edge on! The selection criteria have to be chosen with some care, if one wants to obtain a clear picture. We found them in Leiden in the spring of 1984 by using the colours of the IRAS counterparts of $\mathrm{OH}$ maser stars (now called OH/IR stars). Recent $18 \mathrm{~cm}$ observations in Parkes (Australia) and in Effelsberg (BRD) show that more than $\frac{1}{3}$ of the IR sources are indeed $\mathrm{OH}$ masers with the maser line profile characteristic of an expanding shell. Therefore, I am convinced that the fast majority of sources in Figure 1 are similar to $\mathrm{OH} / \mathrm{IR}$ stars: stellar objects in their very last stage of evolution, on the so-called Asymptotic Giant Branch. For the stars near the galactic centre (assumed to be at $8.7 \mathrm{kpc}$ distance) we can estimate the luminosities and we find these to be between 2000 and $6000 L_{\odot}$. The existence of such stars in the disk of the Galaxy is to be expected, but not their existence in the bulge: the point is that the brighter stars (say $L>4000 L_{\odot}$ ) are estimated to originate from Main-Sequence masses $>1.1 M_{\odot}$. Unless these stars are metal rich (say $Z \gtrsim 0.10$ ), they cannot be as old as stars in the bulge are supposed to be, i.e., 15 Gyr. So the question appears to be: is the bulge old, but metal rich; or young? Clearly this question has been asked before and has been tentatively answered - for example by Arp in 1965 (Astrophys. J. 141, 43). But I doubt whether any earlier picture has shown the problem better defined than Figure 1. An analysis of Figure 1 is at present being made in Leiden by Wil van der Veen, who will write a thesis about it (ready in 1988?). A proper analysis not only covers the question: "what stage of 
evolution is this of what type of star', but also the question: 'what does their presence in the Bulge tell us about the formation of the Bulge'. To us it is an exciting task to find answers to these questions.

\section{Discussion}

P. G. Mezger: Did you have to correct any of your earlier statements about the distribution, number of stars, etc., on the basis of IRAS observations? We think that there are more those stars in the Galaxy with a distribution different for their luminosities.

H.J. Habing: I do not expect that there will be more than 10000 or 15000 in the Galaxy. Their total luminosity will be relatively small, about $10^{8}$ solar luminosities. 